\title{
S-Adenosyl-L-Methionine Improves the Changes of Calcium Content and Glucose Metabolism after Transient Ischemia in the Rat
}

\author{
Yoshiki MATSUI, Ikuko YAMAGAMI and Nobuyoshi IWATA \\ Biological Research I aboratories, Sankyo Co., Ltd., \\ Hiromachi 1-2-58, Shinagawa-ku, Tokyo 140, Japan
}

Accepted October 7, 1988

\begin{abstract}
Effects of S-adenosyl-L-methionine on $\mathrm{Ca}$ accumulation as well as the changes of electrolytes contents and glucose metabolism after transient ischemia were investigated. In the 4-vessel occlusion model of rats, brain $\mathrm{Ca}$ content increased to $200-250 \%$ of that in sham operated rats 1 day after 60 min transient ischemia. The change in the striatum was more severe than that in the cortex or hippocampus. Na content increased and $\mathrm{K}$ content decreased, and glucose, pyruvate and lactate contents increased significantly in the striatum 1 day after transient ischemia. SAM $(100 \mathrm{mg} / \mathrm{kg}$, i.p.) was injected at the end of occlusion and every hour for $5 \mathrm{hr}$ thereafter. Treatment with SAM reduced $\mathrm{Ca}$ accumulation, decrease of $K$, and increase of lactate and pyruvate in the striatum significantly. The present results suggest that the inhibition of $\mathrm{Ca}$ accumulation might partly explain the beneficial effects of SAM on the cerebral dysfunction following ischemia.
\end{abstract}

S-Adenosyl-L-methionine (SAM), which plays an important role in transmethylation as a methyl donor, is well known to have various effects. For example. SAM affects the membrane fluidity $(1,2)$, monoamine metabolism (3) and exocytosis (4). In addition to these experiments in vitro, the effect of SAM has been also studied in vivo for clinical application. Caruso et al. (5) showed the effectiveness of SAM as an antidepressant, and Stramentinoli (6) showed the improvement of galactosamine induced liver injury by SAM. Recently, Trovarelli et al. (7) showed that SAM improved the changes of lipid metabolism induced by ischemia, and Matsui et al. (8) and Sato et al. (9) showed the protective effect of SAM against neuronal degeneration induced by transient ischemia. Furthermore, Takahashi et al. (10) showed that SAM facilitated recovery from behavioral disturbances due to brain damage. Improvement of edema (11) and energy metabolism (12) by SAM after transient ischemia were also reported. These observations suggest the usefulness of SAM in the treatment of cerebrovascular disease.
Many reports showed that ischemia disrupted the $\mathrm{Ca}$ homeostasis in the cell $(13,14)$ and suggested that $\mathrm{Ca}$ triggers a reaction following ischemia. Dienel et al. (15) showed that ischemia-induced neuronal death, socalled delayed neuronal death, is related to $\mathrm{Ca}$ metabolism and accumulation. We therefore examined the effects of SAM on the changes of $\mathrm{Ca}, \mathrm{Na}$ and $\mathrm{K}$ content and also on the changes of glucose metabolites after transient ischemia in order to study the beneficial effect of SAM on the brain dysfunction induced by ischemia.

\section{Materials and Methods}

Preparation of transient ischemic model: Male Wistar rats (260-310 g) were used. Transient ischemia was prepared by the method of Pulsinelli and Brierley (16) with a slight modification (8). In brief, vertebral arteries were electrocauterized under sodium pentobarbital (35 mg/kg, i.p.) anesthesia, and 3 days after, carotid arteries were exposed under halothane anesthesia ( $4 \%$ in air). The arteries were occluded with small clips after recovery from anesthesia. Animals showing 
a loss of righting reflex and rigidity of forelimbs during the ischemia were used for the present experiment. The clips were removed after 30 or $60 \mathrm{~min}$ and reflow of blood was verified visually. Surgical incision was closed and rats were allowed to recover. SAM (SAM$\mathrm{HCl}$ salt; Fuji Chemical Industry Co., Ltd.; $100 \mathrm{mg} / \mathrm{kg}$ as free form of SAM, i.p.) was injected just after reperfusion and every hour for $5 \mathrm{hr}$ successively. In sham operated animals, vertebral arteries were electrocauterized and carotid arteries were exposed as described above but not occluded.

Determination of glucose metabolites: Rats were killed by a microwave applicator ( New Japan Radio Co., Ltd., NJE 2601 type, 4 kW. $2.2 \mathrm{sec})$, and the brain was removed. The cerebral cortex $(\mathrm{Cx})$, hippocampus (Hip) and striatum (St) were dissected out and homogenized with $10 \mathrm{vol}$. of $0.4 \mathrm{~N}$ perchloric acid (containing $1 \mathrm{mM}$ EDTA). After centrifugation at $3000 \mathrm{~g} \times 10 \mathrm{~min}$, the supernatants were neutralized with $5 \mathrm{M}$ potassium carbonate. The samples were again centrifuged at 10000 $\mathrm{g} \times 20 \mathrm{~min}$, and the supernatants were stored at $-80^{\circ} \mathrm{C}$ unitil determination of glucose metabolites. ATP, creatine phosphate and glucose metabolites were determined by the methods of Lowry and Passonneau (17).

Determination of electrolytes: Rats were killed by decapitation and brains were removed. Cx. Hip and St were homogenized with 10 vol. of distilled water. An aliquot was used for protein determination, and $500 \mu \mathrm{l}$ of the homogenate was ashed with conc. $\mathrm{HNO}_{3}$ and conc. perchloric acid. The residue was dissolved in $2.5 \% \mathrm{SrCl}_{2}$ solution. $\mathrm{Ca}, \mathrm{K}$ and $\mathrm{Na}$ were determined by atomic absorption.

\section{Results}

Most of the animals recovered the righting reflex within $3 \mathrm{hr}$ after $60 \mathrm{~min}$ transient ischemia. Spontaneous movements and the response to handling in the operated animals were lower than those in sham operated rat. Convulsions were observed in 6 of 24 rats treated with saline and in 2 of 10 rats treated with SAM during 3 days after reperfusion. In these behavioral parameters, no significant differences were observed between the SAM and saline treated groups.

Table 1 shows the mortality rate of animals with transient forebrain ischemia. Half of the animals with 60 min transient ischemia could not survive for 3 days. However, when animals were treated with SAM after transient ischemia, all animals were still alive 3 days

\section{Ca increase after $60 \mathrm{~min}$ transient ischemia}

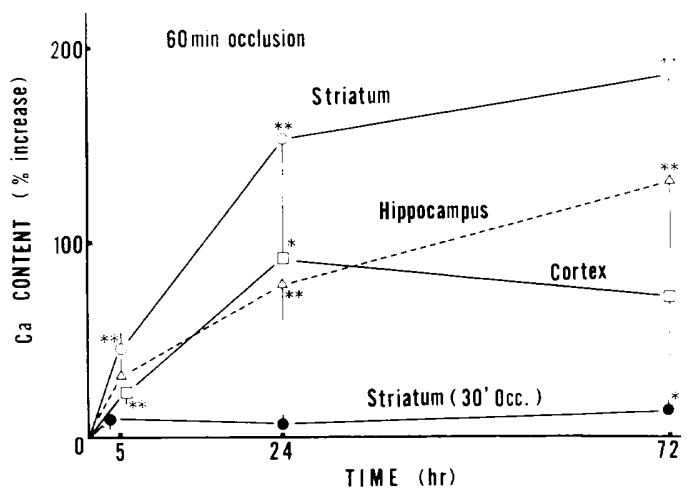

Fig. 1. Changes of brain $\mathrm{Ca}$ content after transient ischemia. Animals with 30 or 60 min transient ischemia were operated on, and then killed at the time indicated after reperfusion. Ca contents in 3 regions were determined as shown in Materials and Methods. Ca contents in the sham operated group were $0.326 \pm$ $0.013(n=15), 0.355 \pm 0.007(n=15)$ and $0.339 \pm$ $0.013(n=15)$ in St, Hip and Cx, respectively $(\mu \mathrm{g} / \mathrm{mg}$ protein). ${ }^{*} \mathrm{P}<0.05,{ }^{*} \mathrm{P}<0.01$ (Student's $t$-test vs. sham operated group). Each point represents the mean \pm S.E. $(n=5-12)$.

Table 1. Effect of SAM on the mortality rate after transient ischemia. Animals with transient ischemia were operated on and the number of animals that died were counted 1 or 3 days after reperfusion

\begin{tabular}{ccc}
\hline & \multicolumn{2}{c}{ Mortality rate $^{* *}$} \\
\hline Saline & $\frac{1}{1 \text { day }^{*}}$ & 3 days $^{*}$ \\
SAM & $0 / 24$ & $9 / 18$ \\
\hline
\end{tabular}

The mortality rate at 1 day or 3 days after reperfusion was calculated from separate experiments. ${ }^{*}$ Days after reperfusion. ${ }^{* *}$ No. of animals died/total No. of operated animals, 
after reperfusion $(P<0.05$, by chi square analysis).

Figure 1 shows the changes of $\mathrm{Ca}$ content after transient ischemia. Ca content in St increased slightly $14 \%$ of the control 3 days after $30 \mathrm{~min}$ transient ischemia and in the other two regions, $\mathrm{Ca}$ content also increased to the same degree as that in St (data were not shown). When the ischemia was prolonged to $60 \mathrm{~min}$. Ca content in the 3 regions increased significantly $5 \mathrm{hr}$ after reperfusion and continued to increase up to $24 \mathrm{hr}$ after reperfusion. Three days after reperfusion, $\mathrm{Ca}$ contents in the animals still alive remained at a high level.

Since most of the animals could survive and $\mathrm{Ca}$ contents increased markedly $24 \mathrm{hr}$ after $60 \mathrm{~min}$ transient ischemia, the effect of SAM on the increase of $\mathrm{Ca}$ content was studied 24 $\mathrm{hr}$ after reperfusion. Figure 2 shows that SAM reduced the increase of $\mathrm{Ca}$ content in $\mathrm{St}$

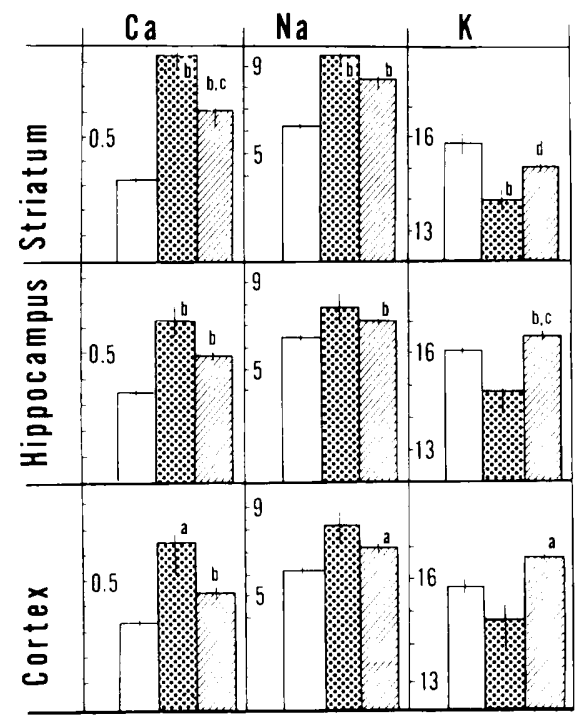

Fig. 2. Effects of SAM on electrolyte contents $(\mu \mathrm{g} /$ $\mathrm{mg}$ protein) 1 day after reperfusion. Animals with 60 min transient ischemia were operated on and killed 1 day after reperfusion. The electrolyte contents were determined as shown in Materials and Methods. Open columns: sham operated, dotted columns: ischemic, and hatched columns: ischemia + SAM treated group. Each column represents the mean \pm S.E. $\quad(n=7-15)$. a: $P<0.05, \quad$ b: $P<0.01$ (Student's $t$-test vs. sham operated group). c: $\mathrm{P}<$ 0.05 , d: $P<0.01$ (Student's $t$-test vs. ischemic group). significantly, and a similar tendency was observed in $\mathrm{Cx}$ and Hip, but not significantly. At 1 day after reperfusion. $\mathrm{Na}$ content increased and $\mathrm{K}$ content decreased significantly in St, but electrolyte changes in the other 2 regions were not statistically significant. Treatment with SAM improved the decrease of $K$ content in St and Hip.

The effect of SAM on the changes of $\mathrm{Na}$ content was not statistically significant in the 3 regions.

The changes of ATP, creatine phosphate and glucose metabolites were studied in the rat brain 1 day after 60 min transient ischemia.

ATP content did not change in the 3 regions, but creatine phosphate increased in $\mathrm{Cx}$ as shown in Fig. 3. Glucose, pyruvate and lactate increased in the 3 regions, and the increase in St was particularly marked. Figure 3 also shows the effect of SAM on the changes of lactate, pyruvate and glucose content 1 day after reperfusion. When rats were treated 6 times with SAM after reperfusion, the increase of pyruvate and lactate were inhibited significantly in St, but not Hip and Cx. A trend toward inhibition was also observed in the increase of glucose. The treatment of SAM did not affect the content of ATP and creatine phosphate in the rat after the transient ischemia.

\section{Discussion}

Various changes have been observed during and after brain ischemia, for example, catabolism of phospholipids $(18,19)$, membrane degradation (20), disturbance of mitochondrial function $(21,22)$ and cell death (23). It is well accepted that these changes are closely related to $\mathrm{Ca}$ mobilization $(13,15$, 24). In the present experiments, 60 min transient ischemia increased $\mathrm{Ca}$ content in the brain. This result is in line with the observation by Ophoff et al. (25). who observed the accumulation of $\mathrm{Ca}$ after transient ischemia in the cat, and also in line with the result by Dienel (15), who found that the Ca accumulation in St is more severe than that in other regions.

Delayed neuronal death was observed in 30 min occlusion and reperfusion in a previous study (8). However, transient ischemia for 30 min could not produce sufficient in- 

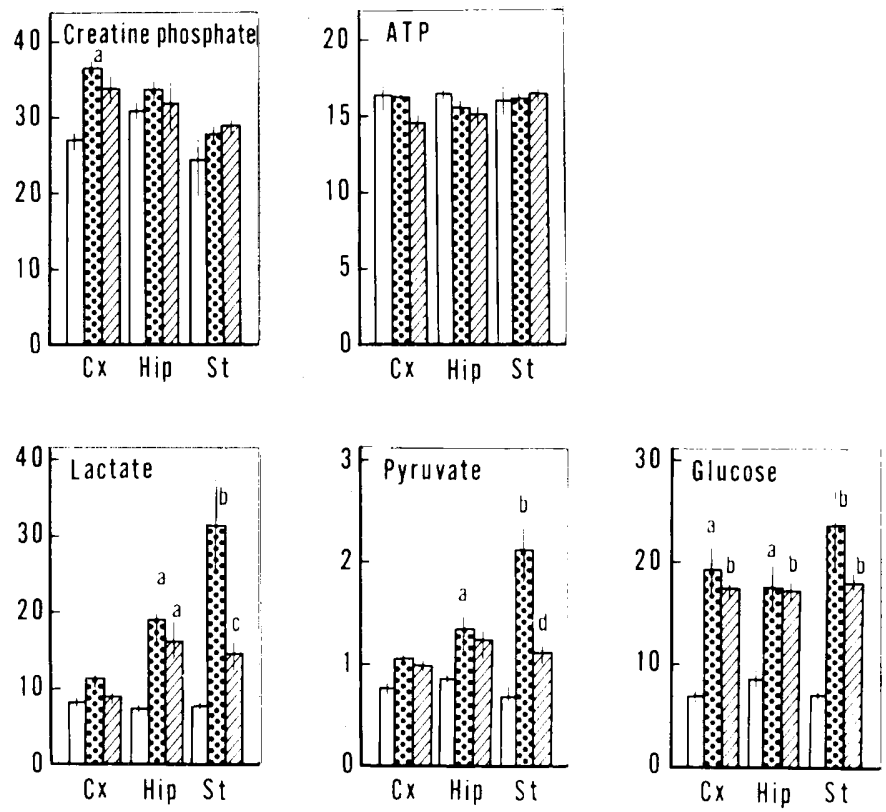

Fig. 3. Creatine phosphate. ATP and glucose metabolite contents in 3 brain regions (Cx: cortex, Hip: hippocampus, St: striatum) in sham (open column), ischemic (dotted column) and ischemia+SAM treated (hatched column) groups. Animals with 60 min transient ischemia were operated on and killed 1 day after reperfusion. Creatine phosphate. ATP and glucose metabolites were determined as shown in Materials and Methods. Each column represents the mean ( $\mu$ moles/mg protein) $\pm S . E$. $(n=8-9)$. a: $P<0.05$, b: $P<0.01$ (Student's $t$-test vs. sham operated group). $\quad c: P<0.05, d: P<0.01$ (Student's $t$-test vs. ischemic group).

crease of $\mathrm{Ca}$ content to study the effect of SAM. Therefore, we prolonged the occlusion period to $60 \mathrm{~min}$ and observed a marked increase of $\mathrm{Ca}$ content and also changes of glucose metabolism and electrolyte content 1 day after reperfusion. The changes of glucose metabolites and electrolyte contents in St were more severe than that in the other 2 regions. This difference between the regions can not be explained now.

When the animals were operated in $60 \mathrm{~min}$ transient ischemia, half of the animals died within 3 days after reperfusion. Therefore, it appears that transient ischemia in this condition was very severe. Sato et al. (26) and and Mabe et al. (27) reported the improvement of EEG pattern after transient ischemia by SAM, and therefore the improvement of behavioral changes after transient ischemia by SAM was also expected. However, SAM improved the mortality rate, but not spontaneous movement, occurence of convulsion and the recovery of the righting reflex after transient ischemia in the present experiment. The lack of beneficial effect of SAM on behavioral changes after transient ischemia may be due to the severe condition in this experiment.

One day after $60 \mathrm{~min}$ transient ischemia, increase of $\mathrm{Ca}$ content, changes of electrolyte and glucose metabolism were observed, but most animals survived and ATP content recovered to the normal level, and so we studied the effect of SAM on these changes 1 day after transient ischemia.

Treatment with S.AM after transient ischemia reduced the $\mathrm{Ca}$ accumulation by $26 \%, 23 \%$ and $30 \%$ of that in the ischemic group in St, Hip and $C x$, respectively. Although the effect on the changes in $C x$ and Hip were not statistically significant because the variations were large in the ischemic groups, the results that the inhibition of $\mathrm{Ca}$ accumulation in three regions were approximately the same indicates that SAM inhibited $\mathrm{Ca}$ accumulation in the brain induced 
by transient ischemia. Ishikawa et al. (28) showed that SAM inhibited mitochondrial swelling induced by $\mathrm{Ca}$ or phospholipase $A_{2}$ and inhibited phospholipase directly, and phospholipase was reported to be activated by $\mathrm{Ca}$ (29). Taking these observations in the in vitro experiments into consideration, it is likely that SAM might mitigate mitochondrial dysfunction to improve glucose metabolism or inhibit the phospholipase activation to prevent membrane degradation, and then finally protect against cell death via inhibition of $\mathrm{Ca}$ accumulation.

The mechanism of the inhibitory effect of SAM on $\mathrm{Ca}$ accumulation remains to be explained. The possibility is not excluded that the primary effect of SAM is not inhibition of $\mathrm{Ca}$ accumulation but facilitation of phospholipid metabolism $(7,30)$ to prevent membrane damage and leakage of $\mathrm{Ca}$, because perturbation of glucose and phospholipid metabolism, and changes of ionic flux following transient ischemia are related and closely affect each other (13).

The mechanism of the effect of SAM on the deficits following ischemia should be studied in further detail. However, it is clear that the various beneficial effects of SAM suggest its usefulness in treating cerebrovascular disease and that part of its beneficial effects seems to be due to the inhibition of $\mathrm{Ca}$ accumulation induced by transient ischemia.

\section{References}

1 Hirata, F. and Axelrod, J.: Enzymatic synthesis and rapid translocation of phosphatidyl choline by two methyltransferase in erythrocyte membranes. Proc. Natl. Acad. Sci. U.S.A. 75, 23482352 (1978)

2 Hirata, F. and Axelrod, J.: Enzymatic methylation of phosphatidylethanolamine increases erythrocyte membrane fluidity. Nature 275, 219220 (1978)

3 Cursio, M., Catto, E., Stramentinolli, G. and Algeri, S.: Effect of S-adenosyl-L-methionine on serotonin metabolism in rat brain. Prog. Neuropsychopharmacol. 2, 65-91 (1978)

4 Crews, G.L., Morita, Y., Hirata, F., Axelrod, J. and Siraganian, P.P.: Phospholipid methylation affects immunogloblin E-mediated histamine and arachidonic acid release in rat leukemic basophilis. Biochem. Biophys. Res. Commun. 93, 42-49 (1980)
5 Caruso, I., Fumaglli, M., Boccasini, L., Puttini, P.S., Ciniselli, G. and Cavarlli, G.: Antidepressant activity of S-adenosylmethionine. Lancet 1, 904 (1984)

6 Stramentinoli, G., Gualano, M. and Ideo, G.: Protective role of $\mathrm{S}$-adenosyl-L-methionine on liver injury induced by D-galactosamine in rat. Biochem. Pharmacol. 27, 1431-1433 (1978)

7 Trovarelli, G., DeMedio, G.E., Porcellati, S., Stramentinoli, G. and Porcellati, G.: The effect of $\mathrm{S}$-adenosyl-L-methionine on ischemia-induced disturbances of brain phospholipid in the gerbil. Neurochem. Res. 8, 1597-1609 (1983)

8 Matsui, Y., Kubo, Y. and Iwata, N.: S-Adenosyl$\mathrm{L}$-methionine prevents ischemic neuronal death. Eur. J. Pharmacol. 144, 211-216 (1987)

9 Sato, H., Hariyama, M. and Moriguchi, K.: SAdenosyl-L-methionine protects the hippocampal CAl neurons from the ischemic neuronal death in rat. Biochem. Biophys. Res. Commun. 150, 491-496 (1988)

10 Takahashi, J., Nishino, $H$. and Ono, T.: SAdenosyl-L-methionine facilitates recovery from deficits in delayed response and hand movement tasks following brain lesions in monkeys. Exp. Neurol. 98, 459-471 (1987)

11 Kozuka, M., Kubo, Y., Kobayashi, K. and Iwata, N.: Effects of S-adenosyl-L-methionine upon ischemia-induced brain edema in Mongolian gerbils and spontaneously hypertensive rats. Japan. J. Pharmacol. 46, 225-236 (1988)

12 Sato, M., Tobita, M., Ohtomo, H., Izumiya, M. and Kogure, K.: Effect of S-adenosyl-L-methionine on the cerebral energy metabolism and microcirculation in the rat subjected to transient forebrain ischemia. Brain and Nerve 39, 11511156 (1987)

13 Siesjo, B.K.: Cell damage in the brain: A speculative synthesis. J. Cereb. Blood Flow Metab. 1, 155-185 (1981)

14 Farber, J.L.: The role of calcium in cell death. Life Sci. 29, 1289-1295 (1981)

15 Dienel, G.A.: Regional accumulation of $\mathrm{Ca}$ in postischemic rat brain. J. Neurochem. 43, 913925 (1984)

16 Pulsinelli, W.A. and Brierley, J.B.: A new model of bilateral hemispheric ischemia in the unanesthetized rat. Stroke 10, 267-272 (1979)

17 Lowry, O.H. and Passonneau, J.V.: A Plexible System of Enzymatic Analysis. Academic Press, New York (1972)

18 Bazan, N.G., Jr.: Effects of ischemia and electroconvulsive shock on free fatty acid pool in the brain. Biochim. Biophys. Acta 218, 1-10 (1970)

19 Cenedella, R.J., Galli, C. and Paoletti, R.: Brain 
free fatty acid levels in rats sacrificed by decapitation versus focused microwave irradiation. Lipids 10, 290-293 (1975)

20 Schwartz, J.P. Mrusulja, B.B., Mrusulja, B.J., Passonneau, J.V. and Klatzo, I.: Alterations of cyclic nucleotide related enzymes and ATPase during unilateral ischemia and recirculation in gerbil cerebral cortex. J. Neurochem. 27, 101107 (1976)

21 Ozawa, K., Seta, K., Araki, H. and Handa, H.: The effect of ischemia on mitochondrial metabolism. J. Biochem. 61, 512-514 (1967)

22 Hillered, L., Siesjo, B.K. and Arfors, K.E.: Mitochondrial response to transient forebrain ischemia and recirculation in the rat. J. Cereb. Blood Flow Metab. 4, 438-446 (1984)

23 Kirino, T. and Sano, K.: Fine structural nature of delayed neuronal death following ischemia in the gerbil hippocampus. Acta Neuropathol. 62, 209-218 (1984)

24 Hossmann, K.-A., Ophoff, B.G., SchmidtKastner, R. and Oschlies, U.: Mitochondrial calcium sequestration in cortical and hippocampal neurons after prolonged ischemia of the cat brain. Acta Neuropathol. 68, 230-238 (1985)

25 Ophoff, B.G., Hossmann, K.-A., Bodsh, W. and Paschen, W.: Relationship between regional calcium content and energy metabolism during recovery from prolonged cerebral ischemia. Monogr. Neural Sci. 11, 22-27 (1984)

26 Sato, H., Nakano, M., Horita, I. and Takahashi, J.: Effect of S-adenosyl-L-methionine sulfate tosylate (FO-1561) on functional recovery after transient cerebral ischemia in rats. Folia Pharmacol. Japon. 90, 91--95 (1987) (Abs. in English)

27 Mabe, H., Ohara, S. and Nagai, H.: Effects of FO-1561 on postischemic cerebral functional and metabolic recovery in experimental cerebral ischemia. Brain and Nerve 38, 1087-1090 (1986)

28 Ishikawa, Y., Kanzaki, T., Morisaki, N., Saito, Y. and Yoshida, S.: Inhibitory effect of FO-1561 (S-Adenosyl-L-methionine sulfate tosylate) on phospholipase $A_{2}$. Tohoku J. Exp. Med. 153, 211-215 (1987)

29 Borgeat, P. and Samuelsson, B.: Arachidonic acid metabolism in polymorphonuclear leukocytes: effects of ionophore A.23187. Proc. Natl. Acad. Sci. U.S.A. 76, 2148-2152 (1979)

30 Strittmatter, W.J., Hirata, F. and Axelrod, J.: Increased $\mathrm{Ca}^{2+}$-ATPase activity associateo with methylation of phospholipid in human erythrocytes. Biochem. Biophys. Res. Commun. 88, $147-153$ (1979) 